\title{
Complete genome sequence of a versatile hydrocarbon degrader, Pseudomonas aeruginosa DN1 isolated from petroleum-contaminated soil
}

Wen Dong, Chunqiu He, Yanpeng Li, Chao Huang, Fulin Chen, Yanling Ma*

Shaanxi Provincial Key Laboratory of Biotechnology, Key Laboratory of Resources Biology and Biotechnology in Western China, Ministry of Education, College of life science, Northwest University, Xi'an, Shaanxi 710069, China;

* Corresponding author

Yanling Ma

College of Life Science, Northwest University, 229 Taibai North Rd, Xi'an, ShaanXi 710069, China;

Phone: (86) 029-88303572. Fax: 029-88302411. Email: mayanling@nwu.edu.cn 


\begin{abstract}
Pseudomonas aeruginosa DN1 was isolated from a petroleum-contaminated soil from Changqing Oilfield with its capability to degrade high molecular weight polycyclic aromatic hydrocarbons (HMW PAHs) and crude oil. Herein, the whole genome sequence analysis of $P$. aeruginosa strain DN1 was reported, consisting of a size of 6,641,902bp chromosome assembled genome (67.09 mol\% $\mathrm{G}+\mathrm{C}$ content) and a $317,349 \mathrm{bp}$ plasmid assembled genome (57.01 mol\% G+C content). According to the genome information, strain DN1 encodes various genes related to degradation of aliphatic hydrocarbons and aromatic compounds. In addition, DN1 contains gene clusters for biosynthesis and regulation of biosurfactant rhamnolipids. These genes may serve as a basis of further elucidation of the genetic background of this promising strain, and provide insights into investigating the metabolic and regulatory mechanisms of hydrocarbon biodegradation.
\end{abstract}

\title{
Key words
}

Complete genome sequence; Pseudomonas aeruginosa strain DN1; Hydrocarbon biodegradation; Synthesis of rhamnolipids 


\section{Introduction}

Hydrocarbon pollutions have significant environmental consequences and present substantial hazards to human health and destroy the ecological balance, which may take years or even decades to recover. Numerous remediation technologies have been developed for the removal of hydrocarbon contamination, including physic-chemical and biological methods. Among these, bioremediation is one of effective clean-up strategies implemental for the restoration of hydrocarbon polluted environment due to its cost effectiveness, less stress to environment and complete recovery of contaminated sites(Kanaly and Harayama, 2010; Hu et al., 2013). Bacterial strains able to degrade hydrocarbons are known to be ubiquitous in nature. However, bioremediation efficiency can be limited by the bioavailability of hydrocarbons and survival of bacteria under adverse crude oil contaminated environment (Nikolopoulou and Pasadakis, 2013; Trellu et al., 2016). The isolation of indigenous bacteria is often beneficial since they can produce oil dispense compounds like biosurfactants that could accelerate hydrocarbon degradation (Ayed et al., 2015).

A number of microorganisms have potential on bioremediation of hydrocarbon contaminated environment(Lazar et al., 1995; Seo et al., 2009). Among these, Pseudomonas aeruginosa is well-known rhamnolipid producer, it is also considered to be ubiquitous hydrocarbon-degrader(Sajna et al., 2015). The type strain, DN1 was isolated from petroleum-contaminated soil in Shaanxi, China, and is already known to degrade various hydrocarbons as the sole carbon. Additionally, the strain DN1 had the ability of producing biosurfactant rhamnolipids in hydrocarbons rich culture medium(Ma et al., 2016). A phylogenetic tree, constructed by MEGA software (version 6.0) and neighbor-joining method, showed a distinct branch of DN1 with various $P$. aeruginosa strains, and strain DN1 was identified as the closest evolutionary relative of $P$. aeruginosa PAO 1 (Fig. 1). In order to gain insights into the genetic bases in its regulatory mechanisms for the further bioremediation study of hydrocarbon contaminants, we sequenced the whole genome of strain DN1, which was presented in this paper. 


\section{Data description}

The whole genome sequencing of strain DN1 was performed using a combined strategy of PacBio RSII sequencing and Illumina Miseq paired-end sequencing platform with long-insert (10 kb) and short-insert (400bp) libraries, respectively. A total of 277038 reads were produced by the PacBio RS II sequencing. The quality of these reads was assessed by the FastQC tool, and the clean reads was de novo assembled by Celera Assembler. Gene prediction was carried out with the Glimmer 3.0 (Delcher et al., 1999) and gene annotation was performed using the NCBI Prokaryotic Genome Annotation Pipeline. The complete strain DN1 genome was composed of a circular $6,641,902 \mathrm{bp}$ chromosome and a circular $317.349 \mathrm{bp}$ plasmid. The chromosome harbored 6,215 open reading frames (ORFs), 64 tRNAs and 16 rRNAs with a GC content of $67.09 \%$. The plasmid consisted of 469 open reading frames (ORF) with a GC content of 57.01\%. Amongst the identified CDSs, 6185 were in the chromosome, and 264 were in the plasmid, respectively (Table1). A total of 5451 of the identified genes were classified to functional categories based on clusters of orthologous genes (COG) designation(Powell et al., 2014): 716 genes for general function prediction only, 549 genes for amino acid transport and metabolism, 495 genes for transcription, 353 genes for inorganic ion transport and metabolism, 327 genes for energy production and conversion, 326 genes for signal transduction mechanisms, 252 genes for cell wall/membrane/envelope biogenesis, 245 genes for lipid transport and metabolism, 226 genes for carbohydrate transport and metabolism, 198 genes for secondary metabolites biosynthesis, transport and catabolism, 196 genes for posttranslational modification and protein turnover and chaperones, 194 genes for translation and ribosomal structure and biogenesis, 185 genes for coenzyme transport and metabolism, 173 genes for replication and recombination and repair, 165 genes for intracellular trafficking and secretion and vesicular transport, 149 genes for cell motility, 98 genes for nucleotide transport and metabolism, 70 genes for defense mechanisms, 33 genes for cell cycle control and cell division and chromosome partitioning, 3 genes for chromatin structure and dynamics, 2 genes for RNA 
processing and modification, and 496 genes for function unknown (Fig. 2).

Experimental findings from different research reports revealed that action of hydrocarbon utilizing bacteria on degradation and mineralization depended on the different catabolic genes and enzymes involved in a variety of known bacterial hydrocarbon degradation pathways and expression(Roy, 2013; Roy et al., 2014). For the metabolism of aromatic compounds, two metabolic pathways were well known, one leading to the formation of protocatechuic acid via o-phthalic acid and the other leading to catechol formation via salicylic acid. Subsequently, protocatechuic acid and catechol are degraded by ortho-cleavage and meta-cleavage pathways, respectively (Mishra et al., 2014). Across the genome of the strain DN1, plenty of genes and gene clusters could contribute to the degradation of aromatic compounds, such as catA (catechol 1, 2 dioxygenase, orf02054), pcaG (protocatechuate 3,4-dioxygenase subunit beta, orf00224) and pcaH (protocatechuate 3,4-dioxygenase alpha subunit, or f00226)(Mishra et al., 2014), homogentisate 1,2-dioxygenase ( $h m g A$, orf01281), 2,4'-dihydroxyacetophenone dioxygenase ( $d a d$, orf04509), homoprotocatechuate 2,3-dioxygenase ( $h p c B$, orf04547), benzoate/toluate 1,2-dioxygenase (orf01400, orf02068, orf02070, orf02071), gentisate 1,2-dioxygenase (orf01281, orf02001, orf06111), 4-hydroxyphenylpyruvate dioxygenase (orf06272), 4-hydroxyphenylacetate 3-monooxygenase(hpaA, hpaC, orf04505, orf04506), 4-hydroxybenzoate 3-monooxygenase (pobA, orf00357)(Gibson and Parales, 2000; Cao et al., 2015; Das et al., 2015). Moreover, many other genes were predicted to encode other enzymes involving in degradation of alkane and alkene, such as alkane 1-monooxygenase (alkB1, alkB2, orf02163, orf05286), polyhydroxyalkanoate synthesis protein (phaF, orf07819), 2-oxo-hepta-3-ene-1,7-dioic cid hydratase (hpcG, orf04550), 2,4-dihydroxyhept-2-ene-1,7-dioic acid aldolase (hpaI, orf04551), 2-nitropropane dioxygenase (orf06032) (Smits et al., 2002; Beilen et al., 2003; Hamme et al., 2003; Beilen and Funhoff, 2007). Notably, the key genes involved in degradation of alkane, alkene and aromatic compounds were identified in genome of strain DN1 with comparative genomic analysis of other bacterial genome from NCBI 
database (Table 2). These results provide clues to certify that strain DN1 is a versatile hydrocarbon degrader. Moreover, strain DN1 genome encodes many genes involving in biosurfactant rhamnolipid synthesis, including rhamnosyltransferase (RhlA), glycosyl transferases (RhlB), L-rhamnosyl transferase (RhlC), dTDP-D-glucose 4,6-dehydratase (RmlB), dTDP-4-dehydrorhamnose reductase (RmlD), glucose-1-phosphate thymidylyltransferase (RmlA), dTDP-4-dehydrorhamnose 3,5-epimerase ( $\mathrm{RmlC})$ and phosphomannomutase (AlgC). Additionally, there are many gene regulatory factors related to rhamnolipid production, such as RsaL, RpoN , RpoS, PhoB, DksA, MvfR, PtxR and AlgR (Reis et al., 2011; Dobler et al., 2015; Lovaglio et al., 2015).

The genome information of strain DN1 provides insights into investigating the metabolic and regulatory mechanisms of hydrocarbon biodegradation. The complete genome chromosome and plasmid sequences have been deposited in the DDBJ/EMBL/GenBank database with accession number CP017099 and CP018048, respectively.

\section{Acknowledgments}

This research was supported by the National Science Foundation for Fostering Talents in Basic Research of the National Natural Science Foundation of China (Grant No. J1210063), the National Science Foundation for Young Scientists of China (Grant No. 31000069) and the research project of Shaanxi Provincial Key Laboratory of Biotechnology (16JS108). 


\section{References}

Ayed, H.B., Jemil, N., Maalej, H., Bayoudh, A., Hmidet, N. and Nasri, M., 2015. Enhancement of solubilization and biodegradation of diesel oil by biosurfactant from Bacillus amyloliquefaciens An6. International Biodeterioration \& Biodegradation 99, 8-14.

Beilen, J.B.V. and Funhoff, E.G., 2007. Alkane hydroxylases involved in microbial alkane degradation. Applied Microbiology and Biotechnology 74, 13-21.

Beilen, J.B.V., Li, Z., Duetz, W.A., Smits, T.H.M. and Witholt, B., 2003. Diversity of Alkane Hydroxylase Systems in the Environment. Oil \& Gas Science \& Technology 58, 427-440.

Cao, J., Lai, Q., Yuan, J. and Shao, Z., 2015. Genomic and metabolic analysis of fluoranthene degradation pathway in Celeribacter indicus P73T. Sci Rep 5, 7741.

Das, D., Baruah, R., Sarma Roy, A., Singh, A.K., Deka Boruah, H.P., Kalita, J. and Bora, T.C., 2015. Complete genome sequence analysis of Pseudomonas aeruginosa N002 reveals its genetic adaptation for crude oil degradation. Genomics 105, 182-90.

Delcher, A.L., Harmon, D., Kasif, S., White, O. and Salzberg, S.L., 1999. Improved microbial gene identification with GLIMMER. Nucleic Acids Research 27, 4636-41.

Dobler, L., Vilela, L.F., Almeida, R.V. and Neves, B.C., 2015. Rhamnolipids in perspective: Gene regulatory pathways, metabolic engineering, production and technological forecasting. New Biotechnology.

Gibson, D.T. and Parales, R.E., 2000. Aromatic hydrocarbon dioxygenases in environmental biotechnology. Current Opinion in Biotechnology 11, 236-243.

Hamme, J.D.V., Singh, A. and Ward, O.P., 2003. Recent Advances in Petroleum Microbiology. Microbiology \& Molecular Biology Reviews Mmbr 67, 503-49.

Hu, G., Li, J. and Zeng, G., 2013. Recent development in the treatment of oily sludge from petroleum industry: A review. Journal of Hazardous Materials 261, 470-490.

Kanaly, R.A. and Harayama, S., 2010. Advances in the field of high-molecular-weight polycyclic aromatic hydrocarbon biodegradation by bacteria. Microbial Biotechnology 3, 136-64.

Lazar, I., Voicu, A., Dobrota, S. and Stefanescu, M., 1995. Investigations on potential bacteria for the bioremediation treatment of environments contaminated with hydrocarbons.

Lovaglio, R.B., Silva, V.L., Ferreira, H., Hausmann, R. and Contiero, J., 2015. Rhamnolipids know-how: Looking for strategies for its industrial dissemination. Biotechnology Advances 33, 1715-1726.

Ma, K.Y., Sun, M.Y., Dong, W., He, C.Q., Chen, F.L. and Ma, Y.L., 2016. Effects of nutrition optimization strategy on rhamnolipid production in a Pseudomonas aeruginosa strain DN1 for bioremediation of crude oil. Biocatalysis \& Agricultural Biotechnology 6, 144-151.

Mishra, S., Singh, S.N. and Pande, V., 2014. Bacteria induced degradation of fluoranthene in minimal salt medium mediated by catabolic enzymes in vitro condition. Bioresour Technol 164, 299-308.

Nikolopoulou, M. and Pasadakis, N., 2013. Enhanced ex situ bioremediation of crude oil contaminated beach sand by supplementation with nutrients and rhamnolipids. Marine Pollution Bulletin 77, 37-44.

Powell, S., Forslund, K., Szklarczyk, D., Trachana, K., Roth, A., Huertacepas, J., Gabaldón, T., Rattei, T., Creevey, C. and Kuhn, M., 2014. eggNOG v4.0: nested orthology inference across 3686 organisms. Nucleic Acids Research 42, 231-9.

Reis, R.S., Pereira, A.G., Neves, B.C. and Freire, D.M.G., 2011. Gene regulation of rhamnolipid 
production in Pseudomonas aeruginosa - A review. Bioresource Technology 102, 6377-6384.

Roy, A.S., 2013. Bioremediation of crude oil contaminated tea plantation soil using two Pseudomonas aeruginosa strains AS 03 and NA 108. African Journal of Biotechnology.

Roy, A.S., Baruah, R., Borah, M., Singh, A.K., Boruah, H.P.D., Saikia, N., Deka, M., Dutta, N. and Bora, T.C., 2014. Bioremediation potential of native hydrocarbon degrading bacterial strains in crude oil contaminated soil under microcosm study. International Biodeterioration \& Biodegradation 94, 79-89.

Sajna, K.V., Sukumaran, R.K., Gottumukkala, L.D. and Pandey, A., 2015. Crude oil biodegradation aided by biosurfactants from Pseudozyma sp. NII 08165 or its culture broth. Bioresource Technology 191, 133-139.

Seo, J.S., Keum, Y.S. and Li, Q.X., 2009. Bacterial degradation of aromatic compounds. International Journal of Environmental Research \& Public Health 6, 33-44.

Smits, T.H., Balada, S.B., Witholt, B. and van Beilen, J.B., 2002. Functional analysis of alkane hydroxylases from gram-negative and gram-positive bacteria. Journal of Bacteriology 184, 1733-42.

Trellu, C., Mousset, E., Pechaud, Y., Huguenot, D., Hullebusch, E.D.V., Esposito, G. and Oturan, M.A., 2016. Removal of hydrophobic organic pollutants from soil washing/flushing solutions: $A$ critical review. Journal of Hazardous Materials 306, 149-174. 
Table 1 General genome features of $P$. aeruginosa DN1

\begin{tabular}{lll}
\hline Feature & Chromosome & Plasmid \\
\hline Length (bp) & $6,641,902$ & 317,349 \\
G+C content (\%) & 67.09 & 57.01 \\
Protein coding genes (CDSs) & 6185 & 264 \\
rRNA number & 12 & 0 \\
tRNA number & 64 & 0 \\
ncRNA & 78 & 0 \\
GenBank accession & CP017099 & CP018048 \\
\hline
\end{tabular}


Table 2 Comparison of gene clusters related to degradation of chain hydrocarbon and aromatic compounds in P. aeruginosa DN1 and other $P$. aeruginosa strains retrieved from http://www.pseudomonas.com/

\begin{tabular}{|c|c|c|c|c|c|c|c|c|c|c|}
\hline \multirow[t]{2}{*}{ Strain } & \multicolumn{5}{|c|}{ dioxygenase } & \multicolumn{4}{|c|}{ monooxygenase } & \multirow[b]{2}{*}{ alkB2 } \\
\hline & pcaH & $p c a G$ & catA & $h m g A$ & dad & pobA & hpaA & hpaC & alkB1 & \\
\hline P. aeruginosa $\mathrm{PAO} 1$ & $\bullet$ & $\bullet$ & $\bullet$ & $\bullet$ & & 0 & 0 & 0 & 0 & 0 \\
\hline P. aeruginosa $\mathrm{PA} 7$ & $\bullet$ & $\bullet$ & $\bullet$ & $\bullet$ & & $\circ$ & $\circ$ & $\circ$ & & \\
\hline P. aeruginosa DN1 & • & $\bullet$ & $\bullet$ & • & $\bullet$ & o & $\circ$ & ० & ० & o \\
\hline P. aeruginosa $\mathrm{CI} 27$ & $\bullet$ & $\bullet$ & & $\bullet$ & $\bullet$ & ○ & ○ & ○ & ○ & o \\
\hline P. aeruginosa $\mathrm{CIG} 1$ & • & $\bullet$ & & $\bullet$ & • & o & o & o & ○ & o \\
\hline P. aeruginosa $\mathrm{PAO} 581$ & $\bullet$ & $\bullet$ & $\bullet$ & $\bullet$ & & $\circ$ & $\circ$ & ० & ० & ○ \\
\hline
\end{tabular}




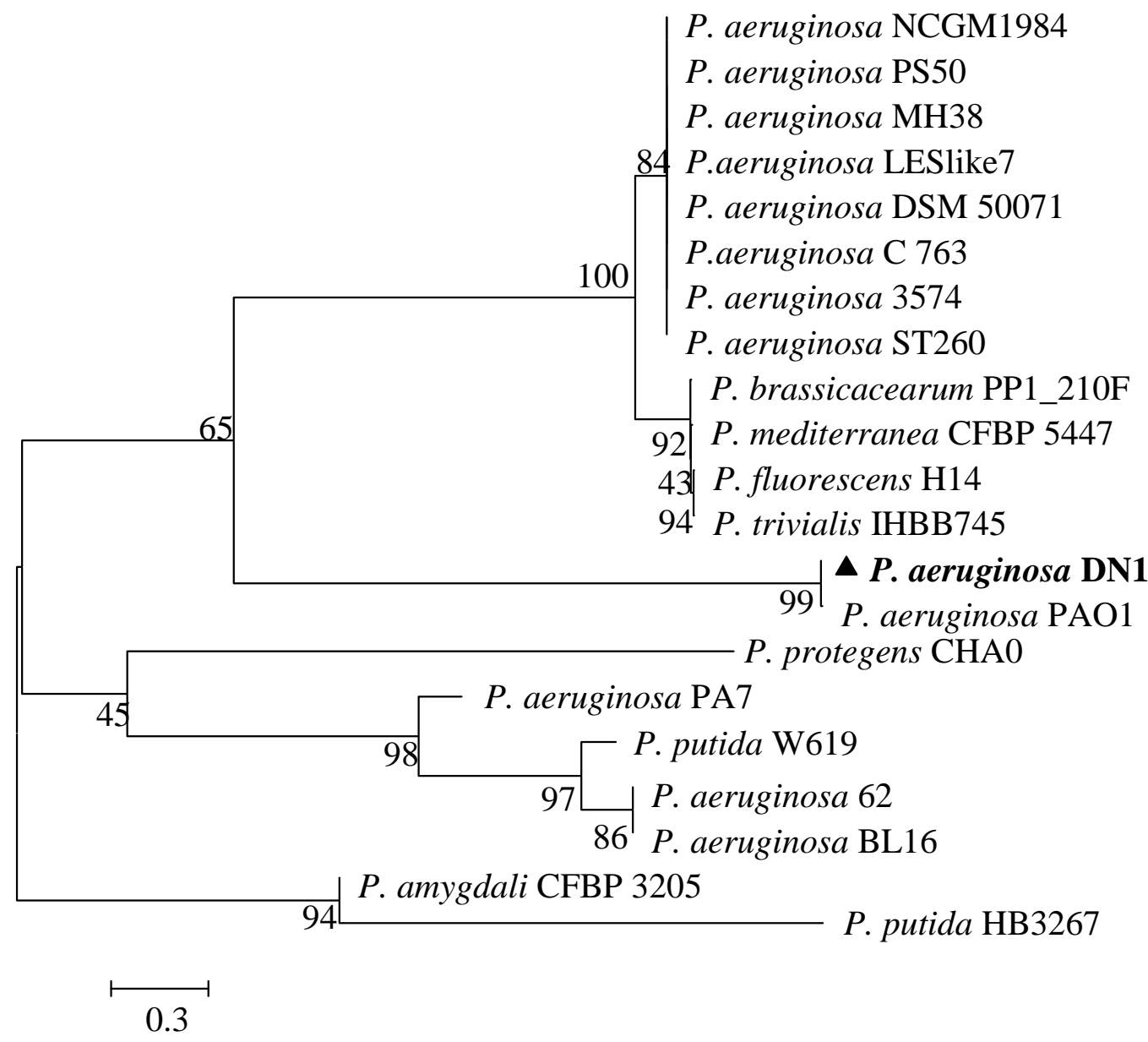

Fig. 1 Phylogenetic tree of Pseudomonas aeruginosa DN1 (The tree was constructed using software MEGA 6.0 by neighbour-joining method based on 16S rDNA gene sequences with 1000 replications in bootstrap test) 


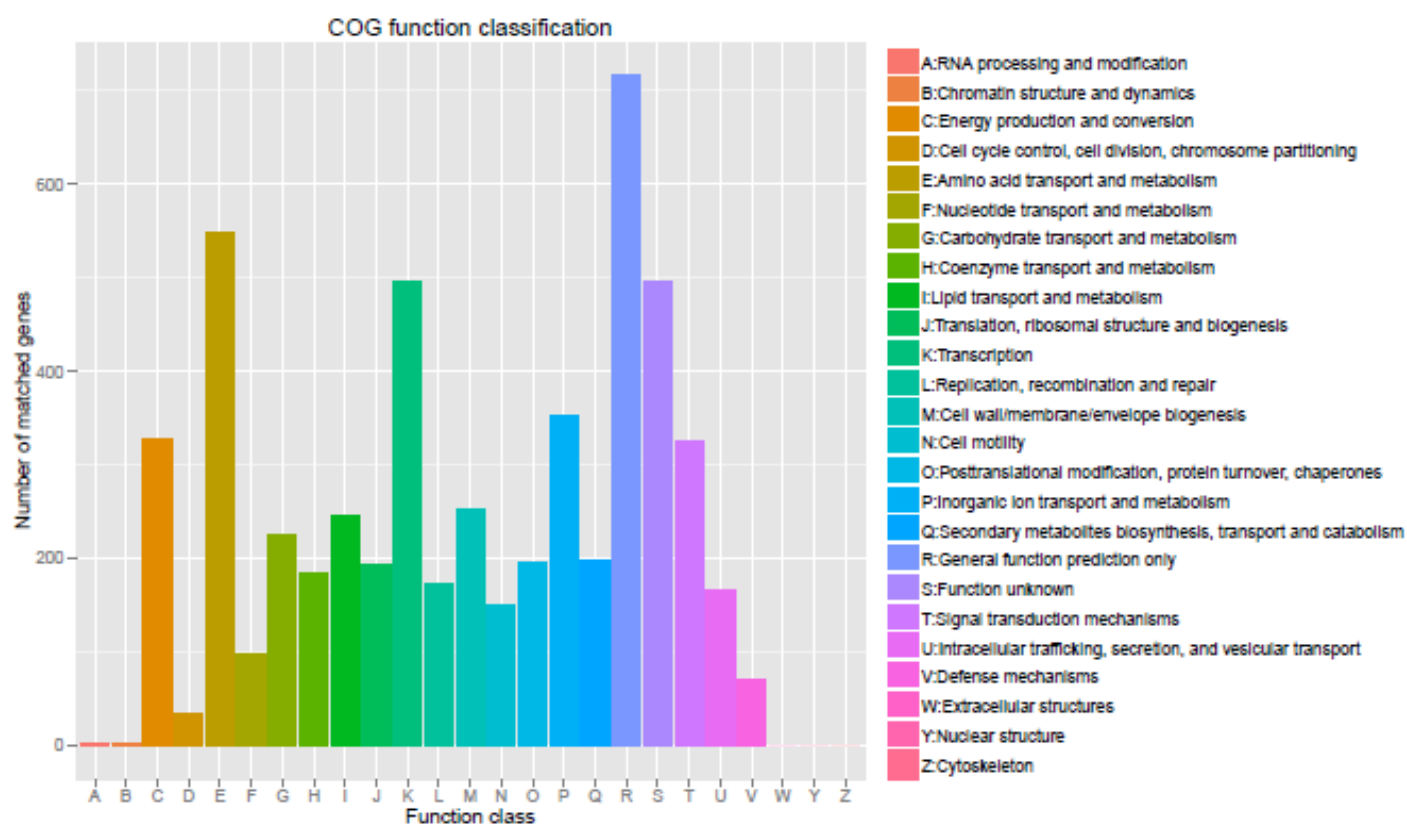

Fig. 2 COG founction classification of $P$. aeruginosa DN1 